\title{
Présences de Spencer dans l'œuvre de Maupassant
}

\section{Antonia Fonyi}

\section{(2) OpenEdition}

Journals

Édition électronique

URL : http://journals.openedition.org/aes/290

DOI : $10.4000 /$ aes.290

ISSN : 2258-093X

\section{Éditeur}

Laboratoire LISAA

\section{Référence électronique}

Antonia Fonyi, «Présences de Spencer dans l'œuvre de Maupassant », Arts et Savoirs [En ligne],

4 | 2014, mis en ligne le 15 mai 2014, consulté le 30 avril 2019. URL : http://journals.openedition.org/ aes/290 ; DOI : 10.4000/aes.290

Ce document a été généré automatiquement le 30 avril 2019.

Centre de recherche LISAA (Littératures SAvoirs et Arts) 


\title{
Présences de Spencer dans l'œuvre de Maupassant
}

\author{
Antonia Fonyi
}

1 L'impact de Spencer sur l'écriture de Maupassant est malaisé à déterminer. Maupassant a lu Spencer, de Spencer. Il possédait Les Premiers Principes ${ }^{1}$ et détenait un temps l' Introduction à la science sociale ${ }^{2}$. Charles Baude de Maurceley, un camarade littéraire de jeunesse, se souvient d'un dîner chez la comtesse de Fleury où Maupassant se mit à parler avec enthousiasme de Spencer qu' "[il] venait [...] de découvrir», mais, "comme les autres invités ne prêtaient qu'une oreille distraite [à ses] propos admiratifs », il prit «le chemin de Mabille $»^{3}$. Cette découverte est impossible à dater. Baude de Maurceley place le dîner à l'époque de la publication de «Boule de suif », donc en 1880 ; seulement, le bal Mabille, situé en effet dans le voisinage de l'hôtel de la comtesse de Fleury, avenue Montaigne, ferma en 1875.

2 Maupassant, toujours selon la même source, dit avoir ignoré auparavant la pensée de Spencer. Pourtant, il semble impossible de distinguer dans sa création un avant et un après la découverte de Spencer. C'est que certaines des idées de ce dernier étaient dans l'air du temps, de sorte que ses lecteurs avaient pris connaissance de sa pensée avant même d'ouvrir un de ses livres. Si Maupassant, à un moment précis, parle de découverte, elle ne concerne fort probablement qu'un domaine particulier du système spencérien. Ajoutons que des idées d'autres philosophes, proches de celles de Spencer, circulaient aussi à la même époque, de sorte qu'il est souvent difficile de distinguer son influence de celle d'un Schopenhauer ou d'un Taine. Encore n'est-il pas toujours sûr, lorsqu'on décèle une ressemblance entre Spencer et Maupassant, qu'elle résulte d'une influence et non d'une rencontre. Dans ces conditions, je préfère parler de la présence de la pensée de Spencer dans l'œuvre de Maupassant, présence plus ou moins nettement délimitée selon le domaine où elle se situe. Je tâcherai d'en montrer le rôle dans trois de ces domaines : les convictions idéologiques, la conception de la connaissance et la logique de la nouvelle. 


\section{Spencer, référence idéologique de Maupassant}

3 Sur le plan idéologique, la présence de la pensée de Spencer dans l'œuvre de Maupassant est évidente et bien connue $e^{4}$. Qu'il suffise donc de la rappeler.

De l'anecdote de Baude de Maurceley, même si son exactitude est discutable, retenons le contraste entre les milieux évoqués : mieux vaut Mabille que la bonne société sourde à Spencer. L'attitude que reflète ce contraste marquera la plupart des nouvelles du " démolisseur $»^{5}$ de préjugés - de valeurs instituées par la norme sociale ou morale - que fut Maupassant. Or Spencer, dans l'Introduction à la science sociale, présente tout un système de préjugés qu'il est nécessaire d'écarter si l'on veut atteindre l'objectivité scientifique, et Maupassant évoque son nom le plus souvent dans les polémiques contre les préjugés.

Deux exemples, pour illustrer le recours à Spencer.

Quel sentiment plus utile au pays que le patriotisme? En est-il un plus élevé, plus noble? Eh bien, moralisateurs [...], allez-vous enseigner aux enfants cette phrase d'un des plus grands penseurs vivants, [...] Herbert Spencer : « Le patriotisme est pour la nation ce qu'est l'égoïsme pour l'individu. $»^{6}$

[Les romantiques] ont substitué l'idée de pardon à l'idée de justice, semant chez nous une sensiblerie miséricordieuse et sentimentale qui a remplacé la raison.

[...]

[...] je trouve que Schopenhauer et Herbert Spencer ont sur la vie beaucoup d'idées plus droites que l'illustre auteur des Misérables. ${ }^{7}$

Il convient de noter, toutefois, que même s'il n'avait pas lu l'Introduction à la science sociale, Maupassant aurait très certainement combattu les préjugés avec énergie. Sur le plan idéologique, l'importance de Spencer résiderait donc moins dans l'influence qu'il aurait exercée sur Maupassant que dans la confirmation que ses thèses apportent aux convictions du polémiste et, surtout, dans le rôle de référence que joue son nom. Attaquer des préjugés, c'est attaquer l'autorité sociale qui les impose. Se référer à Spencer, c'est opposer à cette autorité une autre, forte du prestige dont jouit la philosophie à l'époque. Par cette référence, l'individu Guy de Maupassant, lorsqu'il combat l'égoïsme patriotique ou la sensiblerie promue en morale, se pose en porte-parole d'une pensée philosophique dont la dimension dépasse l'individualité.

7 Il se peut, comme le montre notre deuxième exemple, que Maupassant se réfère en même temps à plusieurs penseurs, notamment à Spencer et à Schopenhauer. Peut-être lui arrive-t-il de confondre quelquefois leurs idées. Aux commentateurs, cela arrive souvent. Je tiens donc à écarter deux erreurs courantes. Contrairement à ce que d'aucuns prétendent, Spencer n'est pas pessimiste ; penseur évolutionniste, il prône les bienfaits de l'éducation et du progrès scientifique. Il n'est pas non plus agnostique dans le sens où il renierait le bien-fondé de la religion; au contraire, tout en posant l'Inconnaissable au fondement même de son système, il cherche à travers toute son œuvre, d'une façon programmatique, à concilier la religion et la science. 


\section{L'Inconnaissable dans l'épistémologie fantastique de Maupassant}

Pour Spencer, l'épistémologie est première, on pourrait même soutenir que l'ontologie s'ensuit. À la base de son système se trouve l'Inconnaissable : ce qui dépasse les limites de notre capacité de connaître, de concevoir. En font partie l'Origine, l'Éternité, l'Infini, l'Absolu, la Matière, la Force, etc. Ils en font partie, sans en être les attributs pour autant, parce que l'Inconnaissable n'est pas un objet, ni une instance, mais une abstraction énigmatique. À ceci près que, par endroits, Spencer l'identifie à une puissance (power) : « Si la religion et la science peuvent se réconcilier, c'est sur ce fait, le plus profond, le plus large et le plus certain de tous : que la puissance dont l'univers est la manifestation pour nous est complètement impénétrable. $\|^{8}$

9 À la lecture d'un tel passage, on se demande si l'Inconnaissable est pure abstraction ou s'il est une puissance qui agit sur notre destin. Qu'il soit l'une ou l'autre, nous n'avons pas de prise sur lui et nous devons admettre son existence. De là, pour nous, des tensions qui s'annoncent intenables, mais que Spencer propose de soulager en introduisant ce modus vivendi qu'est le relatif.

Le Connaissable est de l'ordre du relatif, principalement à deux titres. Premièrement, parce que, étant la manifestation de l'Inconnaissable, il se définit par rapport, en relation avec l'Inconnaissable. Cette relativité fait lien: grâce à elle, les phénomènes qui constituent le Connaissable participent de l'essence inconnaissable. Deuxièmement, comme les phénomènes ne nous sont pas connus tels qu'en eux-mêmes, mais seulement sous certaines conditions, temporelles, spatiales, climatiques, géographiques, etc., dès que ces conditions changent, nos connaissances changent aussi ; par conséquent, elles ne peuvent avoir qu'une valeur relative. Cette relativité est souplesse, richesse : elle permet de concevoir la différenciation toujours croissante qu'apporte l'évolution. Même si elle ne nous donne accès qu'à ce que les objets ont « de particulier, de multiple, de différent, de modifié, de phénoménal ${ }^{9}$ ", elle est " un milieu entre deux extrêmes », entre science et nescience. ${ }^{10}$

11 Maupassant a fort probablement lu cela, mais il a lu aussi d'autres philosophes, et il est difficile de démêler la part de Spencer dans sa conception de la connaissance. Selon certains commentateurs, l'idée de l'insuffisance de l'expérience sensorielle lui est inspirée par Spencer.

Les Sens sont au nombre de cinq, rien que de cinq.

Supposons que l'homme ait été créé sans oreilles; [...] pour lui, l'Univers serait muet [...].

Mais s'il avait reçu en don d'autres organes, [...] doués [...] de [la] propriété de métamorphoser en perceptions nerveuses les actions et les attributs de tout l'inexploré qui nous entoure, combien plus varié serait le domaine de notre savoir et de nos émotions. ${ }^{11}$

On vient de lire La Vie errante, mais on peut trouver des propos semblables dans Sur l'eau, «Le Horla » et ailleurs. Si j'ai cité La Vie errante, c'est que Maupassant y note, au seuil de ces réflexions : « M. Taine a [...] magistralement traité et développé cette idée. » Sa source est Taine, et non Spencer. Dans « Lettre d'un fou ", il cite sur le même sujet Montesquieu, mais on y entend également un écho de Condillac ${ }^{12}$, et on pourrait évoquer aussi des auteurs contemporains, par exemple D'Hervey de Saint-Denys : 
[L'homme est doué] de cinq instruments différents pour percevoir [...]. [...] Est-ce à dire que toutes les propriétés d'un corps nous seront parfaitement connues [...] ? Assurément non. Les objets que nous connaissons sous des rapports au nombre de cinq [...] doivent être vraisemblablement percevables d'une infinité d'autres manières, dont l'ensemble constituerait cette science absolue des choses qui n'appartient qu'au créateur. Il peut donc exister, dans quelque planète, des êtres doués d'appareils sensoriaux si différents des nôtres que la manière dont une seule et même chose serait perçue par eux ou par nous n'offrirait la moindre analogie. ${ }^{13}$

prise sur l'Inconnaissable impliquent une mobilisation des affects. Mais chez Maupassant, leur action est explicite et exacerbée. Dans les chroniques où il reproche, sur un ton nostalgique, au positivisme d'avoir supprimé la dimension mystérieuse de l'univers, l'Inconnaissable apparaît manifestement comme objet de désir.

Chaque jour [les savants] resserrent leurs lignes élargissant les frontières de la science [...]. En deçà, le connu qui était hier l'inconnu ; au-delà, l'inconnu qui sera le connu de demain.

[...]

Eh bien, malgré moi, malgré [...] la joie de cette émancipation, tous ses voiles levés m'attristent. Il me semble qu'on a dépeuplé le monde. On a supprimé l'Invisible. Et tout me paraît muet, vide, abandonné $!^{18}$

La violence du désir est égale à celle du désespoir suscité par l'impossibilité de la satisfaction : « Heureux ceux [...] que ne soulèvent point sans cesse des élans impétueux et vains vers l'au-delà, [...] vers l'immense mystère de l'Inexploré ! ${ }^{19}$

Le chroniqueur tient un discours rationnel, il a pour tâche de réfléchir sur des réalités actuelles, d'élaborer les réactions qu'elles provoquent en lui de façon à pouvoir les partager avec ses lecteurs. Par conséquent, dans les chroniques l'Inconnaissable s'impose pour ce qu'il est par définition : inaccessible pour la connaissance. En revanche, l'auteur 
de fiction est libre de rejeter la contrainte de la rationalité, et cette liberté, qui caractérise en premier lieu la littérature fantastique, permet de satisfaire le désir d'approcher l'Inconnaissable. Mais ce sera une satisfaction à la Pyrrhus : celui qui l'obtient meurt ou finit dans un asile. Aussi le désir s'accompagne-t-il dans ces cas de peur, se mue-t-il en peur. Dans ces conditions, bien que Spencer soit souvent présent dans le fantastique de Maupassant comme inspirateur de contenus intellectuels, sur le plan affectif sa philosophie optimiste et conciliatrice ne laisse pas d'empreinte. Pour expliquer cette contradiction, il convient de déterminer de plus près l'Inconnaissable de Maupassant.

Les affects ambivalents (désir/peur et leurs dérivés), évoluant vers la prédominance des affects négatifs, sont les représentants d'un fantasme inconscient fortement anxiogène qu'on retrouve à la base de toutes les œuvres de Maupassant ${ }^{20}: c^{\prime}$ est une imago de la mère archaïque - située au tout début de la vie -, un milieu biologique qui entoure l'enfant, un corps sans limites, sans forme, sans structure, sans visage. Elle est irreprésentable telle qu'en elle-même, donc invisible, imperceptible - inconnaissable. Elle est une puissance qui règne, absolue, sur la vie et la mort : c'est elle qui a donné la vie et - donc - c'est elle qui la reprendra, en réabsorbant son enfant dans son corps indifférencié. Spencer, rappelons-le, identifie par endroits l'Inconnaissable à une puissance dont le monde est la manifestation. Maupassant parle d'« inconnaissables Puissances » qui exercent sur nous des « influences mystérieuses $»^{21}$ :

Tout ce qui nous entoure, tout ce que nous voyons sans le regarder, tout ce que nous frôlons sans le connaître, tout ce que nous touchons sans le palper, tout ce que nous rencontrons sans le distinguer, a sur nous, sur nos organes et, par eux, sur nos idées, sur notre cœur lui-même, des effets rapides, surprenants et inexplicables ? ?2

Chez Spencer, la perception sensorielle, son efficacité et ses échecs s'inscrivent dans un processus purement intellectuel. Chez Maupassant, l'expérience intellectuelle va de pair avec une expérience corporelle où les "inconnaissables Puissances" agissent sur les " organes» du sujet comme le ferait un corps sur un autre corps. Comme le milieu biologique maternel tout-puissant agit sur l'organisme de l'enfant.

21 L'association de l'Inconnaissable à l'imago de la mère archaïque apparaît clairement dans Le Horla. Assoiffé de lointains inconnus, le héros salue un navire brésilien qui porte leur émissaire invisible, le Horla, et l'invite par ce geste à débarquer chez lui. Désormais, sa vie est souffrance. De malaises en tourments, il en arrive à vouloir tuer le Horla. Mais, pour le tuer, il faut le connaitre. La connaissance adviendra sous la forme d'une hallucination négative : le héros ne voit pas son reflet parce que le Horla se place entre le miroir et lui. Du coup, il peut définir le Horla, sur le mode négatif : c'est un « corps imperceptible ${ }^{23}$, un « corps inconnaissable ${ }^{24}$, dépourvu "de contours nettement arrêtés $»^{25}$, et qui, à l'instar de la mère archaïque, supprime l'identité de l'autre en absorbant son reflet, en assimilant à son être cette preuve de l'identité qu'est le reflet. La catastrophe est inévitable : la première version de l'histoire se termine à l'asile, la seconde par le suicide.

Il arrive que l'association de l'Inconnaissable à l'imago de la mère archaïque apparaisse moins clairement, parce que la psyché se défend du fantasme anxiogène en le refoulant plus profondément. Dans ces cas, la tonalité affective change et les indices textuels qui renvoient au fantasme inconscient sont banalisés. Un groupe de tels indices est constitué par les éléments de l'appareil maternel : le contenant de l'utérus où l'on est d'abord à son aise, mais où l'on finit par se sentir emprisonné; les eaux amniotiques, toutes sortes d'eau, accompagnées de menace de noyade ; le cordon ombilical, des cordes, fils, ficelles qui traînent partout, accompagnés de menace de strangulation. Dans «La Chevelure ", 
par exemple, l'Inconnaissable - «l'Invisible, l'Impalpable, l'Insaisissable, l'Immatérielle Idée ${ }^{26}$ - est associé à une morte, disparue depuis longtemps, que le héros appelle «la

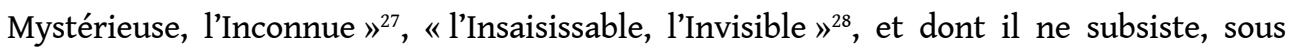
forme matérielle, qu'une natte de cheveux blonds, liés par une corde d'or, un « ruisseau » ${ }^{29}$ de cheveux, une « onde dorée $»^{30}$ : des fils et de l'eau. Le héros parvient à satisfaire son violent désir de posséder la morte - «je l'ai eue $»^{31}$-, et payera sa félicité par l'asile à perpétuité. Cette fois, la peur est absente parce que l'Inconnaissable se trouve transformé en objet d'un désir sexuel qui le pourvoie, sinon de structures, du moins de limites : «j'ai parcouru de mes caresses cette ligne ondulante et divine qui va de la gorge aux pieds $»^{32}$. Mais cette ligne, purement imaginaire, n'est qu'un indice. "Ondulante », elle renvoie à la chevelure métaphorisée comme « onde dorée ", elle-même indice du fantasme de la mère archaïque.

C'est à partir de ce fantasme que s'explique aussi la précarité des frontières chez Maupassant, l'autre point capital sur lequel sa conception de la connaissance diffère de celle de Spencer. On l'aura compris : entre la mère archaïque et l'enfant qu'elle porte les frontières corporelles ne sont pas étanches. D'où la perméabilité des frontières entre le sujet et l'objet : le Horla absorbe celui qui tente de le connaître ; entre l'Inconnaissable et le Connaissable: le Horla se laisse connaitre, ne serait-ce que sur le mode négatif, de même que la morte, «l'Insaisissable, l'Invisible », sur le mode imaginaire : «je l'ai vue, je l'ai tenue, je l'ai eue $»^{33}$.

Nous sommes loin de Spencer. Devant cette instabilité des catégories, on se demande si l'évocation de l'Inconnaissable par Maupassant implique effectivement la présence de la pensée de Spencer à l'arrière-plan de son récit. Mais rappelons que le fantastique de Maupassant se fonde sur le refus de l'épistémè positiviste ("En deçà, le connu qui était hier l'inconnu; au-delà, l'inconnu qui sera le connu de demain »), et c'est ce refus qui motive son adhésion à une philosophie où la conception rationnelle de la connaissance admet le postulat de l'existence de l'Inconnaissable.

\section{Le Connaissable, alias relatif, facteur structurant de la nouvelle réaliste de Maupassant}

Le fantastique, grâce à la très grande liberté dont l'auteur y jouit, est le domaine où l'Inconnaissable et le fantasme anxiogène auquel il s'associe chez Maupassant peuvent apparaître à nu, ou presque. L'auteur de la fiction réaliste, en revanche, doit se soumettre à des contraintes, telles que les lois physiques ou celles d'un système spatio-temporel cohérent ; par là même, il est obligé de recouvrir le fantasme archaïque par un matériel fantasmatique plus tardif - plus évolué - et, partant, plus rassurant. En termes spencériens, la fiction réaliste est le domaine du Connaissable, du conditionné - prise en compte des conditions, temporelles, spatiales, climatiques, sociales, géographiques, etc -, donc, du relatif. Pour Spencer, rappelons-le, le relatif est bénéfique : c'est une catégorie qui permet de saisir le particulier, le multiple, le différent, le monde dans sa richesse évolutive, tout en préservant un rapport à l'Inconnaissable. Maupassant, en revanche, tout en acceptant l'utilité du relatif pour connaître le monde, en déplore l'efficacité limitée.

$[\ldots .$.$] nous nous trompons en jugeant le Connu [...].$

[...] tout est incertain et appréciable de manières différentes.

Tout est faux, tout est possible, tout est douteux. 
Pyrénées, erreur au-delà. »

Et disons : vérité dans notre organe, erreur à côté. ${ }^{34}$

«Vérité en deçà des Pyrénées, erreur au-delà »: relativisme objectif, connaissance déterminée par les conditions externes où l'objet se trouve placé. "Vérité dans notre organe, erreur au-delà»: relativisme subjectif, connaissance déterminée par les propriétés du sujet. Maupassant usera des deux. Bien évidemment, ce n'est pas la lecture de Spencer qui l'incite à avoir recours au relativisme : le genre de dicton « Tout est relatif, c'est le seul absolu ", attribué à Victor Cousin, est monnaie courante à l'époque. Mais par l'usage qu'il fait du relatif, il se laisse rapprocher de Spencer. Il s'en sert, on l'a vu, dans une perspective idéologique ou morale pour battre en brèche des préjugés, mais, dans ces cas, le nom de Spencer est, tout au plus, la caution d'une démarche subversive. Plus importante, de notre point de vue, est la fonction protectrice du relatif qui s'interpose fait lien et séparation à la fois - entre l'Inconnaissable et le sujet connaissant. C'est ainsi qu'il soulage, pour Spencer, les tensions provoquées par l'incapacité du sujet d'avoir prise sur l'Inconnaissable, et c'est le même rôle qui lui sera attribué par la pratique de la nouvelle de Maupassant.

«Le Modèle » est l'histoire d'un peintre et d'une jeune femme, son modèle. Elle devient sa maîtresse, bientôt elle l'ennuie, et il lui annonce qu'il veut reprendre sa liberté. Elle se jette par la fenêtre, se brise les deux jambes, et reste infirme. Il l'épouse.

Le récit suit la progression de la connaissance de l'objet étrange constitué par le couple que forment le peintre et l'infirme. Première explication : « Ceux qui passaient derrière le couple [...] le regardaient d'un air attristé. Toute une légende de dévouement courait. Il l'avait épousée malgré son infirmité, touché par son amour, disait-on. $»^{35}$

C'est une « légende $»^{36}$, une fausse croyance, un préjugé, qui résulte d'un syllogisme fautif.

Prémisse majeure : Tout homme qui épouse une infirme fait acte de dévouement.

Mineure : Jean Summer a épousé une infirme.

Conclusion : Jean Summer fit acte de dévouement.

La majeure est fausse parce qu'elle pose une vérité inconditionnelle, absolue - Tout homme (sous-entendu: en toutes circonstances) qui épouse une infirme fait acte de dévouement -, alors que nous ne pouvons connaître que le conditionné, Spencer et Maupassant sont d'accord sur ce point.

Un ami du peintre se charge de démolir cette légende :

Il l'a épousée... il l'a épousée... comme on épouse, parbleu, par sottise !

[...]

[...] les peintres ont la spécialité des mariages ridicules; ils épousent presque tous des modèles, des vieilles maîtresses, enfin des femmes avariées sous tous les rapports. [...] Après les avoir fait poser, ils les épousent. ${ }^{37}$

Celui qui parle ainsi tient compte de la condition socioprofessionnelle de Jean Summer, de son appartenance à la catégorie des peintres, impliquant un rapport spécifique au mariage. Partant de là, la valeur du mariage de Jean Summer se trouve relativisée, et la légende du dévouement est réfutée.

Mais cette deuxième explication ne tient pas compte de l'infirmité de la jeune femme. Suit alors une troisième explication, l'histoire de l'infirmité racontée par l'ami pourfendeur de légendes, qui a été témoin des événements. Excédé par la bêtise arrogante 
de sa jolie maîtresse, Jean Summer veut la quitter, et avance, comme ultime prétexte, que sa famille veut le marier - voir La Dame aux camélias. Maupassant, «l'Espace » est le lieu où se trouve "embusqué » le «Meurtrier affamé de mort » qu'est Dieu, qui crée pour détruire ${ }^{41}$. Dans « Le Modèle », la jeune femme survit à sa chute dans l'Espace, mais elle restera immobilisée, emprisonnée dans son fauteuil, tout comme le héros du "Horla", lui aussi tenu assis dans un fauteuil par l'envahisseur invisible : «Je désire seulement me lever [...]. Je ne peux pas ! Je suis rivé à mon siège [... ]. $»^{42}$ Tous deux, ils sont repris dans le corps maternel inconnaissable, mais, à la différence $\mathrm{du}$ « Horla », récit fantastique, dans le récit réaliste qu'est « Le Modèle », le fantasme de la mère archaïque ne surgit qu'un instant. Le reste du temps, il est enveloppé, occulté par le Connaissable, mis à distance par le parcours de plusieurs niveaux du relatif.

encours se dessine clairement dans un récit fait par un narrateur, comme c'est le cas $\mathrm{du}$ « Modèle ». Mais on le décèle aussi dans des nouvelles sans narrateur, comme « La Parure ».

C'était une de ces jolies et charmantes filles, nées, comme par une erreur du destin, dans une famille d'employés. Elle n'avait pas de dot [...]; et elle se laissa marier avec un petit commis du ministère de l'instruction publique.

Elle fut simple ne pouvant être parée, mais malheureuse comme une déclassée; car les femmes n'ont point de caste ni de race, leur beauté, leur grâce et leur charme leur servant de naissance et de famille. ${ }^{43}$ malheureuse. C'est la vérité inconditionnée, absolue, qu'il s'agira de réfuter.

Invitée au bal du ministère, Mme Loisel réussit à se procurer une toilette et même une rivière de diamants, prêtée par une amie. Elle est heureuse :

Elle était plus jolie que toutes, élégante, gracieuse, souriante et folle de joie. [...]

Elle dansait avec ivresse, avec emportement, grisée par le plaisir, ne pensant plus à rien, dans le triomphe de sa beauté, dans la gloire de son succès, dans une sorte de nuage de bonheur [...]..$^{4}$ 

qui constitue les effets et, partant, les manifestations de la cause inconnaissable. Selon Maupassant, il nous protège de la satisfaction de notre désir suicidaire d'accéder à l'Inconnaissable. Mais cette valorisation du relatif témoigne-t-elle de la présence de la pensée de Spencer dans l'œuvre de Maupassant? On ne le sait On sait seulement que de semblables pratiques du relatif étaient fort répandues dans les mentalités contemporaines.

51 La présence de la pensée de Spencer dans l'œuvre, à l'arrière-plan de l'œuvre de Maupassant implique des ressemblances. Mais nos lectures ont mis au jour aussi des différences essentielles. La plus importante d'entre elles est celle qui oppose la stabilité des frontières entre catégories chez Spencer à leur instabilité chez Maupassant. La stabilité chez Spencer tient à l'observation rigoureuse du principe logique de la nonidentité : A est égal à $\mathrm{A}$, donc $\mathrm{A}$ n'est pas égal à $\mathrm{B}$ - le sujet ne peut être l'objet, ni l'Inconnaissable être connu en aucune circonstance. Chez Maupassant, au contraire, le Horla peut envahir le sujet à tout moment. Cette rigueur inflexible, voulue, soulignée, assumée par Spencer est probablement une des raisons du désintérêt de la postérité pour son œuvre: notre époque reconnaît d'autres logiques avec celle du tiers exclu. Maupassant le devinait, et c'est peut-être une des raisons de l'intérêt pour son œuvre qui va en se renforçant depuis un demi-siècle.

Mais en est-il vraiment ainsi ? La rigueur logique de Spencer était-elle, pouvait-elle être sans faille? À un endroit, Spencer cite William Hamilton qui, lui, cite Milton pour dire que le connu est « conquis sur l'infini vide et sans forme » (« won from the void and formless infinite » $)^{46}$. Le passage suggère l'idée - et c'est cette idée que le philosophe, Spencer ou Hamilton, se défend d'assumer et laisse formuler par le poète - d'une possibilité de compromis, puisque nous avons conquis, pris, gagné (won) le connu sur l'inconnaissable. Ce, grâce au relatif. Donc, grâce au relatif, partiellement, momentanément, A peut être égal à B. Défaut de cuirasse ou ouverture d'esprit, ce point serait passé inaperçu à mes yeux si je n'avais lu Spencer dans l'optique de Maupassant. Partant de ce point dont je me risquerai à dire qu'il lui est commun avec Maupassant, ne pourrions-nous pas lire Spencer autrement, dans un registre adapté à la sensibilité de notre temps ? 


\section{NOTES}

1. André Vial l'affirme d'après une note inédite de Céard, dans Guy de Maupassant et l'art du roman, Paris, Nizet, 1954, p. 129.

2. Voir les lettres à Flaubert du (14 février 1880)] et (début mars 1880), dans Correspondance, éd. Jacques Suffel, Genève, Edito-Service, 1973, t. I, p. 261 et 271.

3. « Guy de Maupassant. Souvenirs personnels », Le Gaulois, 16 juillet 1925.

4. Voir Vial, Maupassant et l'art du roman, op. cit. ; Laurence A. Gregorio, Maupassant's Fiction and the Darwinian View of Life, New York/Washington/..., Peter Lang, 2005 ; Michael Lerner, Maupassant, New York, Braziller, 1975 ; Jean Salem, Philosophie de Maupassant, Paris, Ellipses, 2000.

5. Cf. la chronique " Notes d'un démolisseur », un réquisitoire contre les "Tartufes », Gil Blas, 17 mai 1882.

6. «Pensées libres ", Le Gaulois, 14 décembre 1881. Maupassant cite l'Introduction à la science sociale , Paris, G. Baillière, 1874, p. 222.

7. «Les Soirées de Médan ", Le Gaulois, 17 avril 1880.

8. Spencer, Les Premiers principes, trad. E. Cazelles, $7^{\mathrm{e}}$ éd., Paris, F. Alcan, 1894 [1871], p. 40.

9. Ibid., p. 66.

10. Ibid., p. 66.

11. Guy de Maupassant, La Vie errante, Paris, Ollendorff, 1890, p. 22.

12. Cf. Laurent Dubreuil, De l'attrait à la possession. Maupassant, Artaud, Blanchot, Paris, Hermann, 2003, p. 13.

13. Leon D'Hervey de Saint-Denys, Les Rêves et les moyens de les diriger. Observations pratiques, Amyot, 1867, p. 406-407.

14. Guy de Maupassant, « Lettre d'un fou », Gil Blas, 17 février 1885.

15. Ibid.

16. « Le Horla », Le Horla, Ollendorff, 1887, p. 6-7.

17. Les Premiers principes, op. cit., p. 65.

18. «Adieu mystères ", Le Gaulois, 8 novembre 1881. Bien entendu, ce reproche ne s'adresse pas à Spencer pour qui le progrès scientifique n'implique pas la suppression, ne serait-elle qu'hypothétique, de l'Inconnaissable: "Si nous regardons la science comme une sphère qui s'agrandit graduellement, nous pouvons dire que son accroissement ne fait qu'accroître ses points de contact avec l'inconnu qui l'environne. » (Les Premiers principes, op. cit., p. 13.)

19. «Par delà », Gil Blas, 10 juin 1884.

20. Ailleurs j'ai présenté d'une façon plus développée la problématique qui suit, notamment dans Maupassant 1993, Paris, Kimé, 1993, ou «Le Horla, double indéterminé », dans Le Double. Chamisso, Dostoïevski, Maupassant, Nabokov, dir. Jean Bessière, Paris, Champion, «Unichamp », 1995, p. 91-141.

21. « Le Horla », op. cit., p. 5.

22. Ibid., p. 6.

23. Ibid., p. 62.

24. Ibid., p. 68.

25. Ibid., p. 62 et 63.

26. Toine, Paris, Marpon et Flammarion, s. d. [1885], p. 134.

27. Ibid., p. 145.

28. Ibid., p. 146.

29. Ibid., p. 143. 
30. Ibid., p. 144.

31. Ibid., p. 145.

32. Ibid.

33. Ibid.

34. « Lettre d'un fou », op. cit.

35. Le Rosier de Madame Husson, Paris, Quantin, 1888, p. 97.

36. Ibid.

37. Ibid., p. 98.

38. Ibid., p. 110.

39. Ibid., p. 101.

40. Ibid., p. 110.

41. Manuscrit, $f^{\circ} 11$, The Harry Ransom Center, The University of Texas at Austin, Artine Artinian Collection, 13.4.

42. «Le Horla », op. cit., p. 46.

43. Contes du jour et de la nuit, Paris, Marpon et Flammarion, s. d. [1885], p. 73-74.

44. Ibid., p. 82.

45. Ibid., p. 90.

46. Spencer, Les Premiers principes, op. cit., p. 66. N. B. La citation (Paradise Lost, Book 3) est extraite d'une évocation de la création du monde par Dieu.

INDEX

Mots-clés : Maupassant (Guy de), inconnaissable, nouvelle, fantastique, réalisme

\section{AUTEUR}

ANTONIA FONYI 